\title{
in honore et exaltatione di Soa Excelentia- Das Standbild des Andrea Doria in der Gestalt Neptuns von Baccio Bandinelli ${ }^{1}$
}

\author{
Katharina Helm
}

Das Standbild des Andrea Doria in der Gestalt Neptuns von Baccio Bandinelli blieb bedauerlicherweise unvollendet und steht heute über einem als Brunnenbecken dienenden Sarkophag auf dem Domplatz in Carrara (Abb. 1). ${ }^{2}$ Es war ursprünglich für Genua geplant, wo stattdessen das Standbild Andrea Dorias als antikisch gerüsteter Feldherr von Giovan Angelo da Montorsoli aufgestellt wurde. Dieser wurde mit der Anfertigung einer neuen Statue beauftragt, nachdem sich abzeichnete, dass Baccio Bandinelli das Monument Neptuns nicht vollenden würde. Doch ist auch dieses Kunstwerk zwar nicht in unvollendetem, so jedoch in unvollständigem Zustand auf uns gekommen: Während der Revolutionsunruhen im Rahmen der Ausrufung der Repubblica Democratica Ligure im Sommer 1797 wurde Montorsolis Statue des Andrea Doria von ihrem Sockel gestürzt und dabei erheblich beschädigt (Abb. 2). ${ }^{3}$ Das lediglich fragmentarisch erhaltene Standbild befindet sich heute im Treppenhaus des Palazzo Ducale in Genua.

Auch wenn die erhaltenen Quellenbelege nicht ausreichend sind, den Grund für den ikonographischen Wechsel in der Konzeption der beiden Statuen zu erhellen, so ist die Diskrepanz der unterschiedlichen Topoi in der Darstellung Andrea Dorias in der Gestalt Neptuns bzw. als Feldherr offensichtlich. Ziel des vorliegenden Beitrags soll daher sein, nach den Funktionen, Zielen und pozentiellen Grenzen der imitatio Neptuni zu fragen, die - abgesehen von den Unstimmigkeiten zwischen Künstler und Auftraggeber - dazu geführt haben könnten, ein zweites, ikonogra-

1 Ich danke Anna Schreurs-Morét und Hans W. Hubert herzlich für ihre Hinweise und die kritische Lektüre des Textes sowie Birgit Laschke-Hubert für eine anregende Diskussion.

2 Die vorliegende Betrachtung der Statue resultiert aus der Beschäftigung der Autorin mit Standbildern von Helden und heroisierten Personen im öffentlichen Stadtraum Italiens und Frankreichs in der Vormoderne, Thema Ihres Dissertationsprojekts im Sonderforschungsbereich 948 .

3 Eine Einführung zur Geschichte dieser beiden Statuen sowie zu ihrer kunsthistorischen Einordnung bietet nach wie vor in übersichtlichster Form H. Keutner, Über die Entstehung und die Form des Standbildes im Cinquecento, in: Münchner Jahrbuch der bildenden Kunst 3, 1956, S. 138-168, besonders S. 143-148. Außerdem P. Boccardo, Andrea Doria e le arti. Committenza e mecenatismo a Genova nel Rinascimento, Rom 1989, S. 105-118; B. Laschke, Fra Giovan Angelo da Montorsoli. Ein Florentiner Bildhauer des 16. Jahrhunderts, Berlin 1993, S. 39-41, S. 153, S. 160; N. Hegener, Divi Iacobi Eques. Selbstdarstellung im Werk des Florentiner Bildhauers Baccio Bandinelli, München 2008, S. 84-85, S. 485-487; B. Laschke-Hubert, Quos ego oder wie der Meeresgott Neptun die Plätze eroberte, in: A. Nova / S. Hanke (Hrsg.), Skulptur und Platz. Raumbesetzung, Raumüberwindung, Interaktion (I Mandorli; 20), Berlin 2014, S. 97-124. 


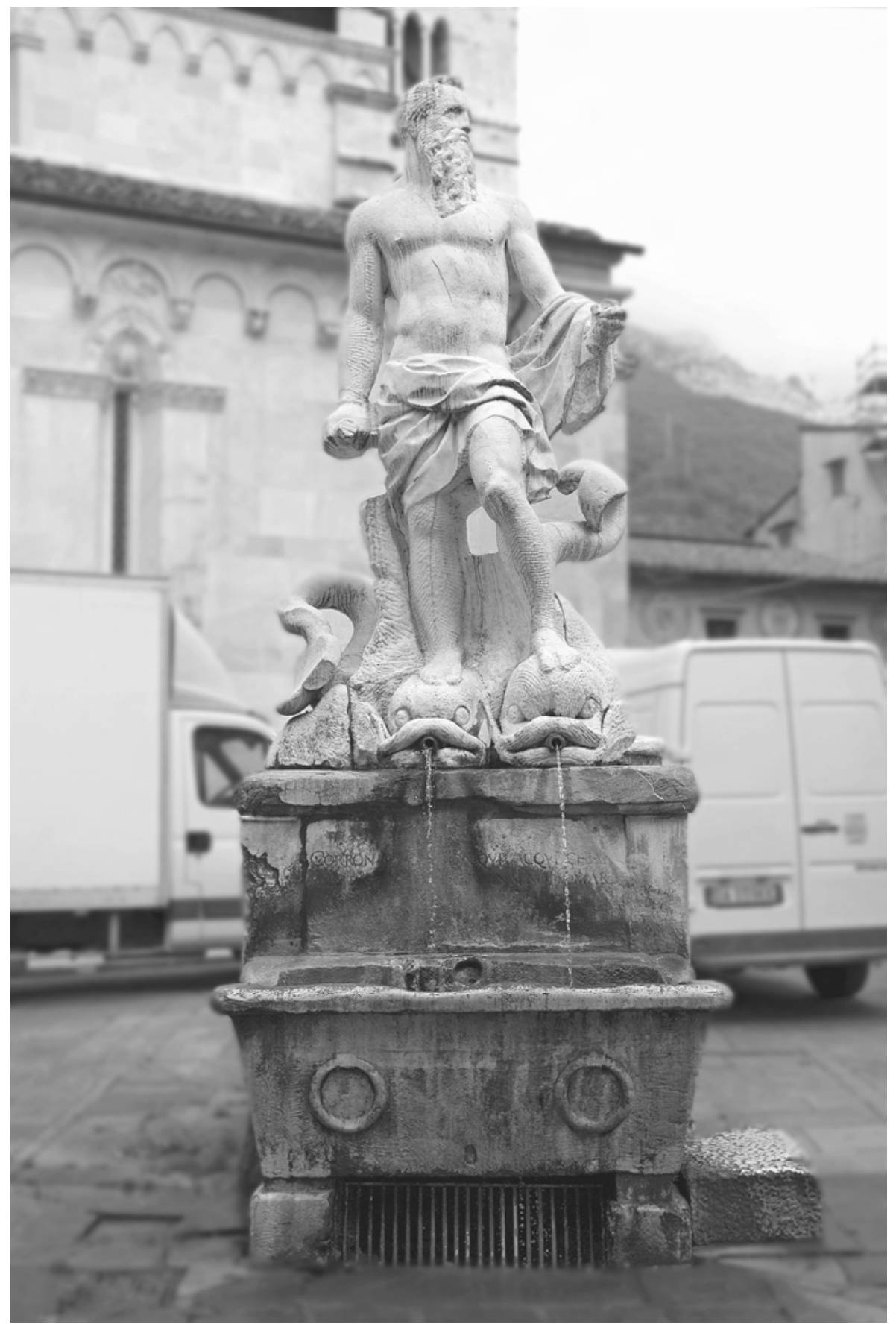

Abb. 1

Baccio Bandinelli, Andrea Doria in der Gestalt Neptuns, 1536-1538, Marmor, Carrara, Piazza del Duomo 


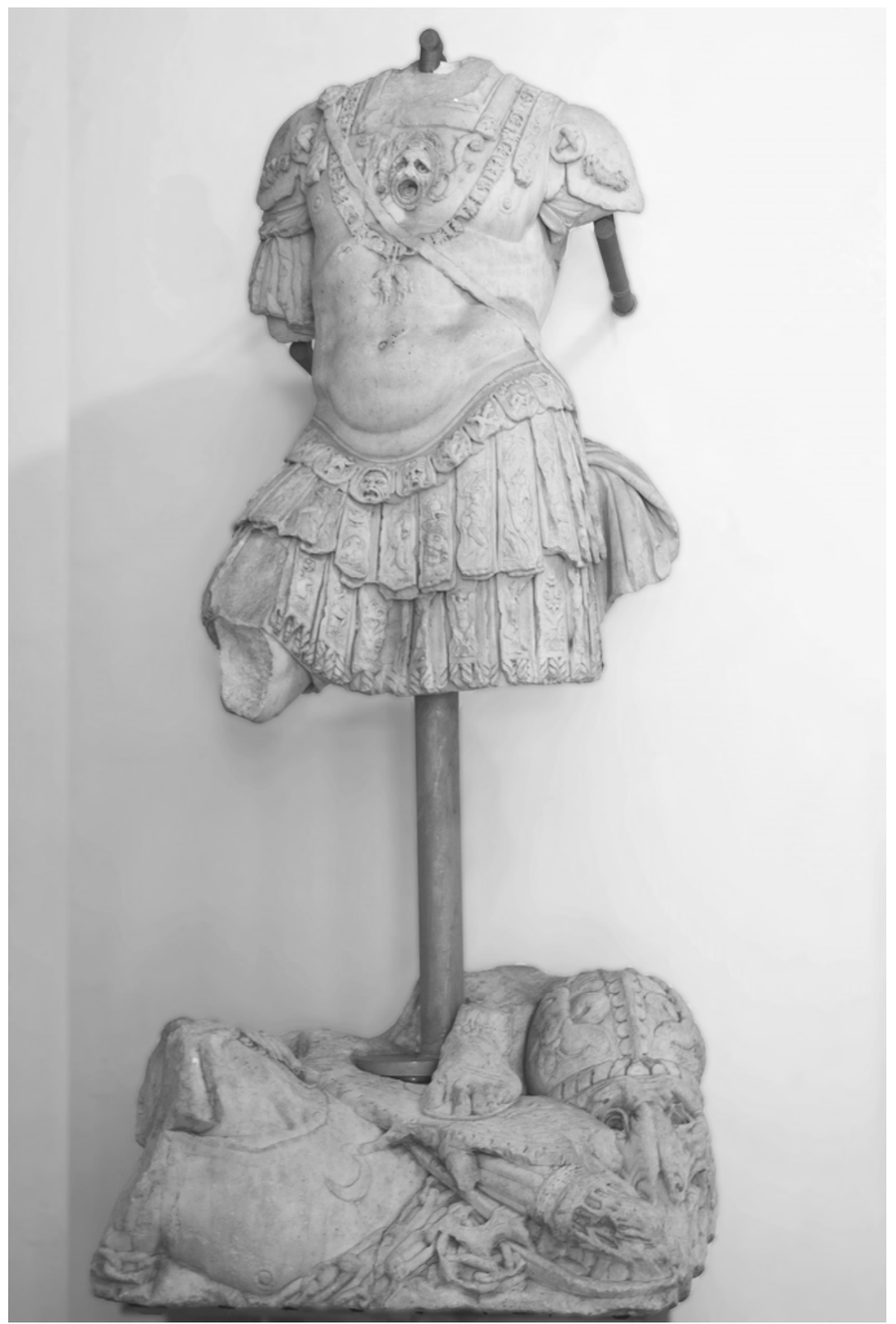

Abb. 2

Giovan Angelo da Montorsoli, Andrea Doria über Türken und Waffengerät, 1539-1540, Marmor, Genua, Palazzo Ducale 
phisch divergierendes Statuenprojekt in Erwägung zu ziehen. Zum besseren Verständnis der Bedeutung der Neptunstatue sei zunächst jedoch erlaubt, den historischen Hintergrund des Auftrages an Baccio Bandinelli knapp zu referieren.

Anlass für die Errichtung des Standbildes Andrea Dorias war die Befreiung Genuas 1528 von der französischen Herrschaft, wodurch eine lange Phase der Fremdbestimmung der ,Superba, wie die ligurische Metropole auch bezeichnet wurde, beendet worden war. ${ }^{4}$ Die Republik war bereits im Mittelalter zu einer bedeutenden Seemacht aufgestiegen und blieb während der gesamten Frühen Neuzeit ein wichtiger Handelsplatz im Mittelmeerraum, wobei sich die genuesischen Handelshäuser zunehmend auf das Bankgeschäft konzentrierten. ${ }^{5}$ Nach der Niederlage gegen Venedig 1381 hatte Genuas Souveränität gegenüber Frankreich und Mailand schleichend abgenommen, so dass die Republik, durch langanhaltende und immer wieder aufflammende interne Konflikte zusätzlich geschwächt, in den folgenden Jahrhunderten zum Zankapfel der um die Hegemonie in Italien ringenden Mächte geworden war. Neben ihrer Stärke als Wirtschafts- und Finanzhandelsstandort führte auch die strategisch günstige Lage der ligurischen Republik dazu, dass Genua eine wichtige Rolle in der Auseinandersetzung zwischen Franz I. und Karl V. um die Vorherrschaft in Italien und um die Kontrolle des Mittelmeerraumes einnahm.

Im Laufe seiner Karriere war der 1466 geborene, aus einer Nebenlinie der alteingesessenen Genueser Familie der Doria stammende Andrea (1466-1560) zunächst als Söldnerführer und später als Flottenkapitän für Innozenz VIII., Guidobaldo da Montefeltro, Alfons und Ferdinand von Aragon, die Republik Genua sowie Franz I. tätig - und stand folglich nacheinander im Sold des Papstes, des Herzogs von Urbino, des Hauses Aragon, der ligurischen Republik und des französischen Königs. ${ }^{6}$ Letzterer ernannte den Genuesen 1525 zum Statthalter der französischen Mittelmeerflotte. Nachdem sich die Beziehung zwischen den beiden jedoch drastisch verschlechtert hatte, entschloss sich Andrea Doria zu einem aufsehenerregenden Frontwechsel und trat im Juli 1528 aus französischen Diensten in das habsburgische Lager über, was ihm zugleich die Ernennung zum Großadmiral des spanischen Königs einbrachte. ${ }^{7}$ Die dadurch entstehende Allianz zwischen Andrea

4 Zur Geschichte Genuas vgl. C. Costantini, La Repubblica di Genova nell'età moderna (Storia d'Italia; 9), Turin 1978; M. Schnettger, „Principe sovrano“ oder „Civitas imperialis“? Die Republik Genua und das Alte Reich in der Frühen Neuzeit (1556-1797), Mainz 2006; A. Pacini, La Genova di Andrea Doria nell'impero di Carlo V (L'officina dello storico; 5), Florenz 1999.

5 Schnettger, „Principe sovrano“ oder „Civitas imperialis“? (Anm. 4), S. 39-40.

6 Zur Person Andrea Dorias vgl. E. Grendi, Andrea Doria, uomo del Rinascimento, in: Atti della Società Ligure di Storia Patria n.s. 19/1, 1979, S. 91-121; J. Heers, Il Principe, il suo tempo, la sua città (Premessa), in: P. Boccardo, Andrea Doria e le arti. Committenza e mecenatismo a Genova nel Rinascimento, Rom 1989, S. 9-15; P. Lingua, Andrea Doria. Principe e pirata nell'Italia del'500, Mailand 1984.

7 S. Hanke, Zwischen Fels und Wasser. Grottenanlagen des 16. und 17. Jahrhunderts in Genua (Tholos Kunsthistorische Studien; 4), Münster 2008, S. 19; E. Grendi, Andrea Doria, uomo del Rinascimento (Anm. 6), S. 100. 
Doria, als dem Vertreter Genuas, und Karl V. ermöglichte dem Habsburger, die iberische mit der österreichisch-burgundischen Reichshälfte zu verbinden und seine machtpolitische Position in Italien zu konsolidieren. Genua wurde als freie Republik anerkannt, stand jedoch unter spanischer Oberhoheit und verzichtete auf eine eigenständige Außenpolitik. Durch die sich erschließenden Handelsverbindungen sowie den Zugriff auf die Finanzgeschäfte der Habsburger gelangte die Republik zu einer neuen wirtschaftlichen Prosperität, welche die Voraussetzung für die daraufhin einsetzende künstlerische Blütezeit Genuas bildete. ${ }^{8}$

Ein weiterer wichtiger Schritt, der die Bedeutung des Großadmirals für die Republik Genua konstituierte, war die von Andrea Doria durchgeführte Reform der Verfassung sowie eine damit einhergehende Neustrukturierung des Staatsapparates. Das neue, strikt oligarchische Regierungssystem schloss alle nichtadeligen Bevölkerungsschichten von jeder politischen Teilhabe aus und versuchte, durch eine engere Zusammenbindung der Familien in 28 neue Familienverbände, sogenannte alberghi, den vormals herrschenden Wettkampf um die Verteilung der politischen Ämter zu eliminieren. ${ }^{9}$ Das höchste Amt in der genuesischen Republik war das des Dogen, dessen Kompetenzen im Vergleich zu jenen vor 1528 deutlich reduziert waren und der sich nach Ablauf seiner zweijährigen Amtszeit einer Beurteilung durch das oberste Kontrollorgan des Staates, die Supremi Sindicatori, unterziehen musste. ${ }^{10}$ Andrea Doria hat sich Zeit seines Lebens zwar nie selbst zum Dogen wählen lassen, muss durch seine Position auf Lebenszeit im Rat der Supremi Sindicatori jedoch zweifelsohne einen recht hohen, wenn nicht sogar einen entscheidenden Einfluss auf die Politik Genuas gehabt haben. ${ }^{11}$

8 Hanke, Zwischen Fels und Wasser (Anm. 6), S. 19.

9 Um Konflikte innerhalb der herrschenden Nobilità zu vermeiden, wurden die Nobili, das heißt alle Familien, die bis 1506/07 an der Regierung partizipiert hatten, in 28 alberghi unterteilt. Alberghi im traditionellen Sinn enger Familienallianzen waren schon seit dem Mittelalter in Genua bekannt, doch diejenigen, die 1528 geschaffen wurden, waren etwas Neues. Den Kern eines jeden albergo bildete eine Familie, die über mindestens sechs case aperte und somit über sechs Familienoberhäupter verfügte. Diesen wurden alle anderen, kleineren Familien zugeordnet, die den Namen der größten Familie eines albergo annahmen, womit jedoch keine rechtliche Gleichstellung einherging. Unter den alberghi verfügten diejenigen Nobili, fortan als ,Vecchi" bezeichnet, die sich von altem Amts- und Feudaladel ableiteten, über eine deutliche Mehrheit von 23 alberghi. Die Popolari oder Nuovi, die sich nicht einer solchen Abstammung rühmen konnten, verfügten über fünf alberghi, vgl. Schnettger, „Principe sovrano“ oder „Civitas imperialis“? (Anm. 4), S. 42-44; Hanke, Zwischen Fels und Wasser (Anm. 6), S. 20.

10 Schnettger, „Principe sovrano“ oder „Civitas imperialis“? (Anm. 4), S. 44.

11 S. Hanke, Die Macht der Giganten. Zu einem verlorenen Jupiter des Marcello Sparzo und der Genueser Kolossalplastik des 16. Jahrhunderts, in: Römisches Jahrbuch der Bibliotheca Hertziana 39, 2009/10, S. 165-186, hier S. 170. Zum möglichen Übereinstimmungsgrad zwischen den Anschauungen Andrea Dorias mit der neuen Verfassung, wie sie die Dodici Riformatori am 11. Oktober 1528 vorlegten, vgl. E. Grendi, La repubblica aristocratica dei genovesi. Politica, carità e commercio fra Cinque e Seicento, Bologna 1987, S. 105-138; Hanke, Zwischen Fels und Wasser (Anm. 6), S. 20. 
Als Würdigung seiner Verdienste wurde Andrea Doria in einem Dekret der Dodici Riformatori vom 7. Oktober 1528 der Titel, pater et liberator patriae verliehen. In demselben Schriftstück wurde außerdem die Errichtung einer Bronzestatue zu Ehren des Admirals und seiner Verdienste für das Vaterland beschlossen.12 Am 20. August 1529, also knapp ein Jahr später, wurde Baccio Bandinelli mit der Ausführung des Kunstwerkes beauftragt. War anfangs noch von einer Bronzestatue für den Ratssaal des Palazzo Ducale die Rede, ${ }^{13}$ so war laut der Vertragsvereinbarung nun eine Marmorstatue beabsichtigt. ${ }^{14}$ Diese sollte, so berichtet Vasari, „[...] un Nettunno in forma del principe Doria, per porsi in su la piazza [...]" darstellen. ${ }^{15}$ Dass mit der überlebensgroßen Neptunstatue tatsächlich Andrea Doria gemeint war, verrät der Blick auf das Porträt des Großadmirals von Bronzino (Farbabb. 2). ${ }^{16}$ Trotz des unvollendeten Zustands des Standbildes lässt sich eine klare physiognomische Übereinstimmung zwischen dem Gemälde und der Skulptur konstatieren. ${ }^{17}$

Es wird deutlich, dass das Statuenprojekt schon zu Beginn wichtigen Veränderungen unterworfen war, die neben dem Materialwechsel von Bronze zu Marmor dazu geführt hatten, dass das Standbild Andrea Dorias aus dem restriktiv zugänglichen Raum des Palazzo Ducale in die Öffentlichkeit des Stadtraumes verlegt wurde. ${ }^{18}$ Überdies ging mit dem Wechsel des Standortes anscheinend auch eine Änderung der Konzeption von einer reinen Porträtstatue Andrea Dorias in eine deutlich überlebensgroße mythologische Darstellungsweise des Admirals einher. Offensichtlich hielt man die für den Ratssaal geplante Ehrenstatue für die Aufstellung im Stadtraum für nicht angemessen, so dass nun ein Standbild mit Dorias Gesichtszügen, jedoch in mythologischer Verkleidung bei Baccio Bandinelli in Auftrag gege-

12 „ut patrie ipsius et pater et liberator dicatur“, zitiert nach L. A. Waldman, Baccio Bandinelli and the Art at the Medici Court. A Corpus of Early Modern Sources (Memoirs of the American Philosophical Society; 251), Philadelphia 2004, Nr. 182, S. 99.

13 „Item ut Ill. ${ }^{\text {mo }}$ D. ${ }^{\text {no }}$ Andree Dorie enea statua in magna Palatii aula quanto ornatius erigi poterit cum nominis inscriptione erigatur", zitiert nach Waldman, Baccio Bandinelli (Anm. 12), Nr. 182, S. 99.

14 „il decto Messer Bartolomeo scultore si obliga al detto Mons. ${ }^{\text {or }}$ Reverendissimo di haver fatta una statua di braccia quattro a similitudine dello Illustrissimo capitano il S. ${ }^{\text {or }}$ Andrea Doria, fornita et con tutte le sue appartenenze, per tutto uno anno dal giorno che lì sarà stato consegnato il marmo in sul luogo ove si debbe lavorare", zitiert nach Waldman, Baccio Bandinelli (Anm. 12), Nr. 197, S. 106.

15 G. Vasari, Opere, Bd. 6, hrsg. von G. Milanesi, Florenz 1906, S. 154.

16 Zum Gemälde Bronzinos vgl. M. Brock, Le portrait d'Andrea Doria en Neptune par Bronzino, in: O. Bonfait / A.-L. Desmas (Hrsg.), Les portraits du pouvoir. Actes du colloque "Lectures du Portrait du Pouvoir entre Art et Histoire », Rome, Villa Médicis, 24-26 avril 2001, Paris 2003, S. 49-63.

17 Die physiognomische Ähnlichkeit wird noch deutlicher, wenn man weitere Bildnisse Andrea Dorias von Porträts und diversen Medaillen, die in jener Zeit entstanden sind, hinzuzieht, vgl. Boccardo, Andrea Doria e le arti (Anm. 3), S. 108-111.

18 Laschke-Hubert, Quos ego oder wie der Meeresgott Neptun die Plätze eroberte (Anm. 3), S. 98-99. 
ben wurde. ${ }^{19}$ Welcher Grund letztlich für die Wahl der Neptunikonographie ausschlaggebend war, ist bis heute ebenso ungeklärt wie der exakte Zeitpunkt, zu dem man sich für das Thema des Meeresgottes entschloss. ${ }^{20}$ Antike Neptundarstellungen waren lediglich in Form von Reliefs von Sarkophagen und von Gemmen her bekannt, vollplastisch gearbeitete Bildwerke sind nicht überliefert. ${ }^{21} \mathrm{Ab} 1525$ wurde Andrea Doria bevorzugt in Anspielung auf seine Tätigkeit als Admiral charakterisiert, woraus sich das Sujet, den Genuesen als Neptun darzustellen, recht gut ableiten lässt. ${ }^{22}$ Die ikonographische Übereinstimmung zwischen dem Gemälde Bronzinos und der Skulptur Bandinellis sowie die Art und Weise, wie in beiden Kunstwerken ein Tuch den unteren Teil des Körpers von Andrea Doria verhüllt, hat dazu geführt, das Porträt in enger Verbindung zu der unvollendeten Statue Baccio Bandinellis zu sehen. ${ }^{23}$ Allerdings lässt die bis heute ungesicherte Datierung des Gemäldes keine Rückschlüsse darauf zu, in welcher zeitlichen Abfolge diese beiden Kunstwerke letztlich entstanden sind. Gleichwohl lässt sich konstatieren, dass sich diese Darstellungskonvention erst mit dem wichtigen Seesieg Andrea Dorias in der Schlacht um Tunis 1535 verfestigte, was nicht zuletzt in der zunehmenden Herstellung von Medaillen mit entsprechendem Bildthema abzulesen ist. ${ }^{24}$ Zudem scheint der Künstler, obwohl er bereits 1529 den Auftrag zur Anferti-

19 Ebd., S. 99.

20 Ebd., S. 99.

21 Grundlegend zur Darstellung Neptuns in Antike und Renaissance vgl. L. Freedman, Neptune in Classical and Renaissance Visual Art, in: International Journal of the Classical Tradition 2, Heft 2, 1995, S. 219-237.

22 Das früheste hier anzuführende Beispiel ist das Porträt Andrea Dorias von Sebastiano del Piombo von 1526, das im Fries am unteren Bildrand Gegenstände zeigt, die alle der Schifffahrt zugeordnet werden können, vgl. Boccardo, Andrea Doria e le arti (Anm. 3), S. 106-108; zur Darstellung Andrea Dorias als praefectus classis und dem möglichen Zusammenhang zwischen dieser Ikonographie und den Gedanken einer renovatio imperii Karls V. vgl. ebd., S. 109.

23 F. Polleroß, Rector Marium or Pater Patriae? The Portraits of Andrea Doria as Neptune, in: L. Freedman / G. Huber-Rebenich (Hrsg.), Wege zum Mythos, Berlin 2001, S. 107-121.

$24 \mathrm{Zu}$ den Medaillen vgl. Boccardo, Andrea Doria e le arti (Anm. 3), S. 108-110. Als Hinweis darauf, dass die Wahl der Neptunikonographie möglicherweise zeitlich mit der siegreichen Schlacht um Tunis zusammenfiel, könnte eine der von Baccio Bandinelli angefertigten Entwurfszeichnungen für das Sockelrelief verstanden werden, die eine Seeschlacht zeigt, vgl. F. Viatte (Hrsg.), Inventaire général des dessins italiens / Musée du Louvre, Département des Arts Graphiques, Bd. 9, Baccio Bandinelli: dessins, sculptures, peinture, Nr. 50, S. 75, S. 161-162; vgl. auch Laschke-Hubert, Quos ego oder wie der Meeresgott Neptun die Plätze eroberte (Anm. 3), S. 99. Zur Gestaltung des Sockels der Neptunstatue Baccio Bandinellis, auf die hier aus Platzgründen nicht eingegangen werden kann, vgl. D. Heikamp, In margine alla „Vita di Baccio Bandinelli“ del Vasari, in: Paragone 191, 1966, S. 51-62; Ders., La fontana di Nettuno. La sua storia nel contesto urbano, in: B. Paolozzi Strozzi / D. Zikos (Hrsg.), L'acqua, la pietra, il fuoco. Bartolomeo Ammanati scultore (Ausstellungskatalog Florenz), Florenz 2011, S. 182-261, hier S. 214; Boccardo, Andrea Doria e le arti (Anm. 3), S. 114; D. Malz, Zwischen Konkurrenz und Bewunderung. Parallelen in der politischen Ikonographie Andrea I. Dorias in Genua und Cosimo I. de’Medici in Florenz, S. 6, http://archiv.ub.uni-heidelberg.de/artdok/volltexte/2012/2149, 30. März 2015; Polleroß, Rector Marium or Pater Patriae? (Anm. 23), S. 115-116; Hegener, Divi Iacobi Eques (Anm. 3), S. 84-85, S. 485-486. 
gung des Standbildes sowie eine erste Zahlung erhalten hatte, abgesehen von der Anfertigung einiger Entwurfszeichnungen und eines Modells in den folgenden Jahren nicht ernsthaft daran gearbeitet zu haben. In einem Brief des Genueser Kanzlers Matteo Senarega aus dem Jahr 1534 wird deutlich, dass dieser damals davon ausging, dass das Statuenprojekt nicht mehr zu retten sei:

„Ho dato cura a un Bartholomeo Bandineli scultor fiorentino di far una statua di marmo di braccia 4 a similitudine dell'Illustrissimo Signor Capitano Andrea Doria in honore et exaltatione di Soa Excelentia, secondo li patti e conditione che V. S. vederà per la copia de la polisa inclusa. E il detto Bartholomeo confessa haver avuto scuti 400 per parte del pagamento de la detta statua, alla quale non è stato mai dato principio né più se ne ragione $[s i c]$ et tutto è andato in fumo [...]." 25

Erst nach mehrfachem Bitten und Drängen vonseiten der Republik, das 1536 in einem in Florenz unter dem Vorsitz Alessandros de' Medici abgehaltenen Schlichtungsgespräch der Parteien gipfelte, scheint sich Baccio Bandinelli ernsthaft dem Genueser Auftrag gewidmet zu haben. ${ }^{26}$

Bedenkt man, dass die Errichtung einer öffentlich zugänglichen Porträtstatue einer noch lebenden Person um 1530 sehr ungewöhnlich und somit potenziell gewisser Akzeptanzschwierigkeiten innerhalb der städtischen Gemeinschaft ausgesetzt war, so liegen die Vorteile einer mythologischen Verkleidung, wie sie der imitatio Neptuni im Standbild von Baccio Bandinelli zugrunde liegt, klar auf der Hand. ${ }^{27}$ Durch die Darstellung als Meeresgott wird die Person Andrea Dorias auf der Grundlage der von ihm errungenen Siege auf See überhöht. Durch die mythologische Verkleidung wird jedoch gleichzeitig die individuelle Erscheinung des Admirals zurückgenommen, wodurch die Präsentation seiner Person im öffentlichen Raum Genuas akzeptabel wird. ${ }^{28}$ Welche Gegenstände der Meeresgott hätte in den Händen halten sollen, lässt sich jedoch nicht vollständig klären. Auf der Entwurfszeichnung Baccio Bandinellis, die im Rahmen der Planungen für die Statue Andrea Dorias entstanden sein muss, trägt Neptun ein Schwert, dessen Griff als Adlerkopf gebildet ist und somit auf das Stemma der Doria und damit auf die Person Andreas verweist (Abb. 3). In der Linken hält er einen Delfin, in der Rechten seinen Dreizack. ${ }^{29}$ Die beiden letztgenannten Attribute der Zeich-

25 Zitiert nach Waldman, Baccio Bandinelli (Anm. 12), Nr. 243, S. 136.

26 Die bei Waldman abgedruckten Quellen verstärken dieses Bild, vgl. Waldman, Baccio Bandinelli (Anm. 12), Nr. 243, Nr. 245, Nr. 250, S. 136-138. Zum Schlichtungsgespräch vgl. ebd., Nr. 256, S. 150-151.

27 Zur zunehmenden Akzeptanz individueller Verherrlichung im Laufe des 16. Jahrhunderts vgl. H. Keutner, Sculpture. Renaissance to Rococo, London 1969, S. 29-30.

28 H. Keutner, Über die Entstehung und die Form des Standbildes im Cinquecento (Anm. 3), S. 148; Laschke-Hubert, Quos ego oder wie der Meeresgott Neptun die Plätze eroberte (Anm. 3), S. 99.

29 Die physiognomische Ähnlichkeit der Zeichnung mit den bekannten Porträts Andrea Dorias wird in der Literatur unterschiedlich bewertet. Denkbar wäre, dass Baccio Bandinelli die Zeichnung angefertigt hat, bevor er Andrea Doria zum ersten Mal sah und das Schwert mit dem Adlerkopf somit der Identifizierung des Admirals gedient hätte. Zur Zeichnung vgl. 


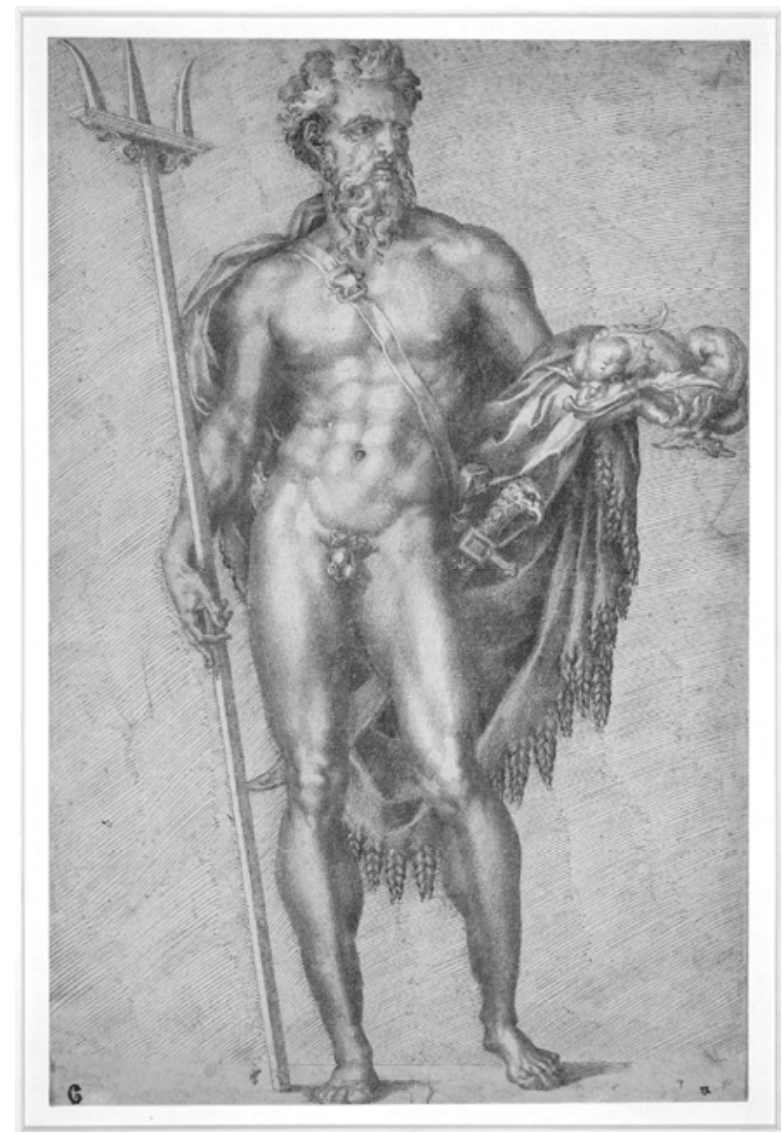

Abb. 3

Baccio Bandinelli, Entwurf für das Standbild Andrea Dorias in der Gestalt Neptuns, um 1529, Feder und braune Tinte auf Papier, 42,5 x 27,5 cm, London, British Museum, Department of Prints and Drawings, Inv. Nr. 1895-9-15-553

nung, Delfin und Dreizack, sind bis zu dem Zeitpunkt, als Baccio Bandinelli die Statue liegen ließ, nur partiell, und zwar in Form der beiden Delfine unter den Füßen Neptuns in die Gestaltung des Standbildes eingeflossen. Jedoch lassen der erhobene linke Arm sowie der in der Hand erkennbare abbozzierte, längliche Gegenstand vermuten, dass die Figur einen Dreizack hätte halten sollen. Über den Gegenstand in der linken Hand des Meeresgottes bleibt zu spekulieren. ${ }^{30}$

R. Ward, Baccio Bandinelli (1493-1560). Drawings from British Collections (Ausstellungskatalog Cambridge), Cambridge 1988, S. 54-56, S. 114; zur Datierung der Zeichnung vgl. D. Heikamp, In margine alla „Vita di Baccio Baccio Bandinelli“ del Vasari (Anm. 24); Ders., La fontana di Nettuno (Anm. 24), S. 212; Boccardo, Andrea Doria e le arti (Anm. 3), S. 114.

30 Durch diese formale Konzeption des Dreizacks in der linken, gemäß der nachwirkenden mittelalterlichen miles christianus-Tradition ,weisen` Hand wäre der Drohgestus des erhobe- 
Neben dieser bildlichen Angleichung wird Andrea Doria in der Biographie von Lorenzo Capelloni aus dem Jahr 1550 - und damit noch zu seinen Lebzeiten auch namentlich an die Figur des Meeresgottes angeglichen: „[...] havendo tante volte solcato il mare, quando il Sole gira i suoi raggi più baßi per questo emispero, tal che dagli huomini sete chiamato il secondo Nettuno." 31

Diese literarische Heroisierung Andrea Dorias, wie sie in der überschwänglichen Rhetorik Lorenzo Capellonis zum Ausdruck kommt, findet im Standbild des Großadmirals als Neptun eine bildliche Präfiguration. Durch die Darstellung in der Gestalt des Meeresgottes werden nicht nur Dorias Leistungen im maritimen Bereich geehrt, sondern er wird gleichzeitig auch als der Seerepublik zugehörig, gleichsam als Sohn der Superba, charakterisiert. Somit tritt zu der personenbezogenen, heroisierenden Funktion der imitatio Neptuni eine kommunal bedeutsame, identitätsstiftende Komponente hinzu. Darüber hinaus wird das Monument durch die Darstellung dieser in der imitatio Neptuni figurierenden Einzelperson, die sich zum Wohl der Republik engagiert, zum Ausdruck eines aufgewerteten Selbstbildes der Genuesen und gleichzeitig zum Sinnbild der von der dargestellten Figur erbrachten Leistung: der neu erworbenen politischen Unabhängigkeit der Republik. Die darin angelegte Vorbildfunktion bezieht sich auf die Tugendhaftigkeit Andrea Dorias, die seine Ernennung zum primus inter pares und in der Folge die Errichtung einer öffentlichen Portätstatue überhaupt erst ermöglichte.

Dieser enge Zusammenhang zwischen den Andrea Doria zugesprochenen positiven Eigenschaften und der Ehrung seiner Person in Form eines von der Republik in Auftrag gegebenen Standbildes wird in einem Brief Pietro Aretinos an den Großadmiral aus dem Jahr 1541 deutlich:

„Ma poiché io debbo pur dirlo, salvete, braccio de la religion di Giesú, core de le imprese sante e flagello de la insolenza infedele. Per la qual cosa tutte le città di battesimo son tenute a consacrarvi la statua, a similitudine di quella che oggi con altera solennità vi consacra Genova: ed è ben dritto, da che voi per propria bontà di natura e per mera generosità d'animo l'avete arricchita d'una libertà perpetua e d'una pace eterna." ${ }^{\text {“32 }}$

nen Dreizacks abgeschwächt bzw. eine friedfertige Haltung des Meeresgottes zum Ausdruck gebracht worden, vgl. H. W. Hubert, Gestaltungen des Heroischen in den Florentiner David-Plastiken, in: A. Aurnhammer / M. Pfister (Hrsg.), Heroen und Heroisierungen in der Renaissance (Wolfenbütteler Abhandlungen zur Renaissanceforschung; 28), Wiesbaden 2013, S. 181-218, hier S. 208, S. 214.

31 Lorenzo Capelloni, Al vittorioso Principe d'Oria, Florenz 1550, o. S. Zur Identifizierung Andrea Dorias mit Neptun wurde in der Forschungsliteratur bereits mehrfach auf einen Brief Paolo Giovios an Clemens VII. aus dem Jahr 1528 hingewiesen, in welchem über die Schlacht von Capo d'Orso bei Salerno berichtet wird. Meines Erachtens ist dort die Bezeichnung als ,Nettuno“ nicht eindeutig Andrea Doria zuzuordnen. Gleichwohl ist von der „gloria del signor messer Andrea Doria" die Rede, dem nicht geschadet werden solle, P. Giovo, Lettere, Bd. 1, 1514-1544, hrsg. von G. G. Ferrero, Rom 1956, S. 118-123, hier S. 119; vgl. auch E. Parma Armani, Il palazzo del Principe Andrea Doria a Fassolo in Genova, in: L'Arte. Rivista di storia dell'arte medievale e moderna n.s. 10, 1970, S. 12-64, hier S. 33-35.

32 P. Aretino, Lettere, Libro Secondo, hrsg. von F. Erspamer, Parma 1998, S. 588. 
Darüber hinaus ist aus dem Brief zu schließen, dass das Ansehen Andrea Dorias nicht nur auf dem von ihm konsolidierten politischen Status der Republik beruht, sondern auch durch den im Zuge der Allianz mit Karl V. erwirkten Frieden, der für Genua eine lange Periode kämpferischer Auseinandersetzungen beendete, begründet wird. Andrea Doria wird somit zum Friedensbringer für sein Vaterland, ein Aspekt, der ebenfalls in der Figur Neptuns angelegt ist, wie Pietro Aretino weiter ausführt:

„Talché vi si debbono gli altari e i tempî sì per i benemeriti sopradetti, sì per la deità attribuitavi da coloro che possono iscorgere in che modo raffrenate i furori dei venti e le tempeste dei mari: con ciò sia che quegli che finser Neptuno per iddio, pronosticorono l'essenza del mirabile vostro avenimento [...]."33

Äußerst aufschlussreich ist in dieser Hinsicht auch Ludovico Ariostos Werk „Orlando Furioso", in welchem Andrea Doria bereits 1532 und somit deutlich früher als in der Biographie Lorenzo Capellonis zwar nicht als Neptun, jedoch als Beschützer, Friedensbringer und zugleich als herausragender Feldherr stilisiert wird. ${ }^{34}$ Im Rahmen der fünf Oktaven, die der Autor den Taten des im Dienst Kaiser Karls V. stehenden Großadmirals widmet, heißt es:

"Come con questi, ovunque andar per terra

si possa, accrescerà l'imperio antico;

così per tutto il mar ch'in mezzo serra

di là l'Europa e di qua l'Afro aprico,

sarà vittorioso in ogni guerra,

poi ch'Andrea Doria s'avra fatto amico.

Questo è quel Doria che fa dai pirati sicuro il vostro mar per tutti i lati.

$[\ldots]$

Sotto la fede entrar, sotto la scorta di questo capitan di ch'io ti parlo, veggio in Italia, ove da lui la porta gli sarà aperta, a la corona Carlo. Veggio che'l premio che di ciò riporta non tien per sé, ma fa a la patria darlo; con prieghi ottien ch'in libertà la metta, dove altri a sé l'avria forse suggetta.

Questa pietà ch'egli a la patria mostra è degna di più onor d'ogni battaglia, ch'in Francia o in Spagna, o nella terra vostra vincesse Giulio, o in Africa o in Tessaglia.

33 Ebd., S. 588-589.

34 Andrea Doria wird mit einzelnen römischen Feldherren verglichen und seine Taten als bedeutungsvoller bewertet, was wiederum im Zusammenhang mit der bereits erwähnten, sich ab 1525 formierenden Darstellungskonvention Andrea Dorias als praefectus classis und somit auch in Verbindung mit dem von Karl V. proklamierten Gedanken der renovatio imperii stehen dürfte, vgl. Boccardo, Andrea Doria e le arti (Anm. 3), S. 109. 
Né il grande Ottavio, né chi seco giostra di pari, Antonio, in più onoranza saglia pe' gesti suoi; ch'ogni lor laude ammorza l'avere usato e la lor patria forza." ${ }^{\text {"35 }}$

Die in der imitatio Neptuni angelegte Semantik des friedenstiftenden Gottes, wie sie auch den Zeilen Pietro Aretinos zu entnehmen ist, geht auf eine recht lange und komplexe Auslegungsgeschichte zurück. ${ }^{36}$ Ausgangspunkt dafür ist eine Textpassage im ersten Buch der „Äneis“ von Vergil, in der Äneas auf dem Weg nach Latium mit seiner Flotte in einen fürchterlichen Sturm gerät, den die zürnende Juno ihm geschickt hatte. Von zentraler Bedeutung ist hierbei das Eingreifen Neptuns, das Vergil folgendermaßen schildert:

„Sprach es, und Tat überholt sein Wort: er sänftigt den Schwall der

Wogen, vertreibt das Wolkengewühl, holt wieder die Sonne.

Triton, voll Eifer, und mit ihm Cymóthoë stoßen vom spitzen

Riff die Schiffe herab. Neptun hilft selbst mit dem Dreizack,

öffnet die mächtigen Wälle von Sand und bändigt die Meerflut, gleitet mit leichten Rädern sodann am Spiegel der Wellen.

Wie es denn so oft geschieht: im Volksgewühle erhebt sich

Aufruhr, wütend rast im Zorn der niedere Pöbel;

Fackeln fliegen und Steine; die Wut schafft Waffen: doch wenn sie dann einen Mann, gewichtig durch frommen Sinn und Verdienste, zufällig sehen, dann schweigen und stehn sie und recken die Ohren.

35 L. Ariosto, Orlando Furioso. Secondo l'edizione del 1532 con le varianti delle edizioni del 1516 e del 1521 (Collezione di opere inedite o rare, pubblicata dalla Commissione per i testi della lingua; 122), Bologna 1960, S. 437, „Wie er, soweit man kommen kann zu Lande, / Das alte Reich durch diese weiterbringt: / So auf dem Meer, das bier von Libyens Sande, / Dort von Europas Ufern wird umringt, / Ist er zu siegen jederzeit imstande, / Wenn er Andrea Doria sich erringt, / Den Doria, der von wilden Räubereien / Wird euer Meer einst überall befreien. / [...] / Von ihm gesichert und von ihm geleitet, / Seh ich den Kaiser nach Italien ziehn, / Wohin Andrea ihm den Weg bereitet / Und wo ihm nun die Krone wird verliehn. / Allein den Lohn, der solche Tat begleitet, / Behält er nicht; dem Staate schenkt er ihn / Und fleht, daß er sein Land befreien dürfe, / Das mancher wohl sich selber unterwürfe. / Die Großmut so uneigennütz'ger Bitten / Verdienet höhern Preis als jede Schlacht, / Die Caesar bei den Galliern, Spaniern, Briten, / In Afrika, in Griechenland vollbracht. / Octavius auch und der mit ihm gestritten, / Antonius, stehn nicht höher in Betracht / Des kriegerischen Ruhms; denn diese dämpften / Ihr Lob dadurch, daß sie ihr Land bekämpften“, Übersetzung: L. Ariosto, Der rasende Roland, Bd. 1, Gesänge 1-25, übers. von J. D. Gries, München 1980, S. 351-352.

36 Grundlegend zur Genese der Figur Neptuns als humanistische Herrscherallegorie sowie den Deutungsprozess der Episode des Quos ego vgl. M. J. Marek, Ekphrasis und Herrscherallegorie. Antike Bildbeschreibungen bei Tizian und Leonardo (Römische Studien der Bibliotheca Hertziana; 3), Worms 1985, S. 75-104; R. Preimesberger, Pontifex Romanus per Aeneam Praesignatus. Die Galleria Pamphilj und ihre Fresken, in: Römisches Jahrbuch für Kunstgeschichte 16, 1976, S. 223-287; zur Deutung der Figur des Neptun als Herrscherallegorie vgl. besonders ebd., S. 271-272; L. Freedman, Neptune in Classical Renaissance Visual Art (Anm. 21), S. 219-237; Laschke-Hubert, Quos ego oder wie der Meeresgott Neptun die Plätze eroberte (Anm. 3), S. 97. 
Er aber lenkt die Erregten durchs Wort, stimmt friedlich die Herzen.

So brach nieder das Tosen der Flut, als über die Wogen

schaute der Vater: er fuhr bei heiterem Himmel und lenkte

fliegend im folgsamen Wagen die Rosse an lockerem Zügel.“37

Bereits durch Vergils eigenen Kommentar, mit dem er die Taten Neptuns erläutert, wird die unter der Bezeichnung, Quos ego ${ }^{6}$ bekannte Episode zu einem allegorischen Bild, das eine rein narrativ-illustrative Auffassung des Motivs von vornherein einschränkt. ${ }^{38}$ Ausgehend von der Deutung Vergils wurde der Meeresgott gegen Ende des Quattrocento zum Bild des idealen Herrschers, der Gerechtigkeit und Milde vereint. ${ }^{39}$ Diese Interpretation Neptuns war jedoch erst mit der eingehenden Deutung der Episode durch Cristoforo Landino im Rahmen seiner „Disputationes Camaldulenses“ aus der Zeit um 1470 möglich. Im neoplatonischen Sinn fasst Landino Vergils „Äneis“ als Allegorie der Seele auf, die sich von allen irdischen Bindungen befreit, um zum Zustand der Kontemplation zu gelangen. ${ }^{40}$ Landino verortet in der Hierarchie der Psyche zwei Hauptinstanzen: die ratio inferior, die der vita activa zugeordnet ist, sowie die ratio superior, die demgemäß der vita contemplativa zugerechnet wird. Die am Anfang der Episode herbeigerufenen Winde stellen nach Auffassung Landinos die unkontrollierbaren Leidenschaften - die ratio inferior - dar, die Neptun - im Sinne der ratio superior - durch sein Eingreifen bändigt und in ihre Grenzen weist. ${ }^{41} \mathrm{Um}$ die hierarchische Stellung der beschriebenen Instanzen der Seele zu verdeutlichen, greift Landino auf ein Gleichnis aus dem Staatswesen zurück und vergleicht die ratio superior mit dem obersten Führer eines Staates. ${ }^{42}$ Neptun wird somit zum Bild einer maßvoll agierenden und reflektierten, ordnenden Kraft, gleichsam zum Symbol der kontemplativen Vernunft. ${ }^{43}$

Vor dem Hintergrund der Interpretation Cristoforo Landinos, die für die nachfolgende Tradition der neoplatonischen „Äneis“-Allegorese im Wesentlichen verbindlich bleibt, wird deutlich, dass sich die Figur Neptuns aufgrund ihres allegorischen Potenzials dazu eignet, um der Person Andrea Dorias die Tugenden eines maß- und friedvollen Herrschers einzuschreiben. ${ }^{44}$ Interessanterweise befand sich die Episode auch als heute leider verloren gegangenes Deckengemälde in einem der beiden repräsentativen Haupträume in Andrea Dorias Palazzo in Fassolo wieder, den dieser ab 1529 durch Perino del Vaga ausmalen ließ. ${ }^{45}$ Dabei bleibt jedoch

37 Vergil, Aeneis, Z. 142-156, Übersetzung: J. Götte (Hrsg.), Vergil, Aeneis, München 1965.

38 Marek, Ekphrasis und Herrscherallegorie (Anm. 36), S. 87.

39 Preimesberger, Pontifex Romanus per Aeneam Praesignatus (Anm. 36), S. 271.

40 Marek, Ekphrasis und Herrscherallegorie (Anm. 36), S. 88.

41 Ebd., S. 89.

42 Ebd., S. 89-90.

43 Preimesberger, Pontifex Romanus per Aeneam Praesignatus (Anm. 36), S. 271.

44 Zur Anwendung des Vergilschen Neptuns auf andere Persönlichkeiten des Cinquecento vgl. ebd., S. 272-275.

45 Nach Aussage Parma Armanis, die sich wiederum auf Vasari bezieht, war dieses Deckengemälde das erste Werk, das Perino del Vaga im Palazzo Doria ausführte, vgl. Parma Armani, Il palazzo del Principe Andrea Doria a Fassolo in Genova (Anm. 31), S. 12-64, hier S. 33. 
unklar, ob sich der Großadmiral selbst oder aber Kaiser Karl V. heroisieren wollte, der 1529 und 1532 im Palazzo Andrea Dorias zu Gast war und in dem Raum, der mit dem Schiffbruch des Äneas ausgemalt war, nächtigte. ${ }^{46}$

Bevor Perino del Vaga die Räume des Palazzo in Fassolo ausmalte, fertigte er Arbeiten für die ephemeren Festapparate an, die anlässlich des feierlichen Einzugs Karls V. in Genua im August 1529 errichtet wurden. ${ }^{47}$ Ein Gemälde, das wahrscheinlich im Durchgang der eintorigen Ehrenpforte hing, die im Hafen stand, veranschaulichte die Befreiung der Stadt durch Andrea Doria. ${ }^{48}$ Aus einem Brief Baccio Bandinellis datierend von Juli 1529 geht hervor, dass der Bildhauer die Errichtung der Festapparate für den Einzug des Habsburgers in Genua selbst gesehen hat. ${ }^{49}$ Angesichts des Vertragsabschlusses bezüglich der Statue des Andrea Doria am 20. August 1529 ist es zudem sehr wahrscheinlich, dass Baccio Bandinelli dem feierlichen Einzug persönlich beigewohnt hat. Außerdem wurde Karl V. im Rahmen seines Einzuges in Bologna am 5. November 1529 mit Darstellungen Neptuns als Herrscher über die Meere gefeiert. ${ }^{50}$ Zusammenfassend lässt sich also feststellen, dass Baccio Bandinelli nicht nur mit den Bildformeln des kaiserlichen Festapparates vertraut war, sondern auch mit der Art und Weise, in welcher die Taten Andrea Dorias bildlich in die politische Kommunikation des Kaisers eingebunden wurden. Außerdem wurde deutlich, dass die Figur Neptuns als Bestandteil imperialer Bildprogramme zwar unterschiedlich ausformuliert wird, jedoch eindeutig dem höfischen Umfeld zugeschrieben werden kann. ${ }^{51}$ Dorit Malz hat bereits auf den Einfluss hingewiesen, den die habsburgische Bildsprache auf die Kunstaufträge der italienischen Herrscherhäuser hatte, sieht in diesen jedoch eher eine Loyalitätsbekundung gegenüber dem Kaiser. ${ }^{52}$ Dass Andrea Doria durch die

46 Malz, Zwischen Konkurrenz und Bewunderung (Anm. 24), S. 20. Zu den Gestalten der antiken Mythologie sowie zur profanen wie auch biblischen Historie als Koordinaten der kaiserlichen Ikonographie vgl. B. Ullrich, Der Kaiser im "giardino dell'Impero“. Zur Rezeption Karls V. in italienischen Bildprogrammen des 16. Jahrhunderts (Humboldt-Schriften zur Kunst- und Bildgeschichte; 3), Berlin 2006, S. 20. Zur engen Beziehung zwischen Andrea Doria und Karl V. vgl. Schnettger, „Principe sovrano“ oder "Civitas imperialis“? (Anm. 4), S. 63.

$47 \mathrm{Zu}$ den Festdekorationen Kaiser Karls V. vgl. M. Philipp, Ehrenpforten für Kaiser Karl V. Festdekorationen als Medien politischer Kommunikation (Kunstgeschichte; 90), Berlin 2011; G. L. Gorse, Entrate e trionfi: cerimonie e decorazioni alla Villa di Andrea Doria in Genova, in: Kunsthistorisches Institut in Florenz (Hrsg.), Disegni genovesi dal Cinquecento al Settecento, Giornate di studio 9-10 maggio 1989, Florenz 1992, S. 9-18.

48 Trotz einer fehlenden Festpublikation zum Einzug in Genua ist das Ereignis durch Quellen hinreichend belegt, vgl. Philipp, Ehrenpforten für Kaiser Karl V. (Anm. 47), S. 112-113.

49 Am Ende seiner Zeilen fügt Baccio Bandinelli einen Satz an, der in keinem kausalen Zusammenhang mit dem Inhalt des Briefes steht und somit als eine Art Bericht über das Geschehen vor Ort in Genua zu verstehen ist: „Qua si fa ponti grandisimi in mare e arhi trionfali per Cieseri.“, zitiert nach Waldman, Baccio Bandinelli (Anm. 12), Nr. 196, S. 105-106. Philipp, Ehrenpforten für Kaiser Karl V. (Anm. 47), S. 113-114.

51 Zum Bild Neptuns im Umfeld der höfischen Kultur vgl. Boccardo, Andrea Doria e le arti (Anm. 3), S. 115.

52 Malz, Zwischen Konkurrenz und Bewunderung (Anm. 24), S. 4. 
Beanspruchung der Neptunikonographie zumindest unterschwellig in ein gewisses Konkurrenzverhältnis zu Karl V. tritt, ist meines Erachtens allerdings nicht gänzlich auszuschließen. Und obwohl bekanntlich das Zerwürfnis zwischen Baccio Bandinelli und den Auftraggebern der ausschlaggebende Grund für das Scheitern des ersten Statuenprojektes gewesen sein dürfte, so lässt sich angesichts des allegorischen Potenzials der Bildnisangleichung Andrea Dorias an den Meeresgott über die Brisanz dieses Konzeptes der besonderen Auszeichnung des Genuesen zumindest mutmaßen. Gleichwohl bleibt festzuhalten, dass durch die imitatio Neptuni vorrangig die Verdienste und Tugenden des Großadmirals sowie seine militärischen Fähigkeiten geehrt werden sollten. Ob jedoch die Interpretation Neptuns als friedvoller Herrscher, die in der Aussagekraft des Standbildes für Andrea Doria zweifellos durchschien, in der Republik einen derart großen Widerwillen erzeugte, dass infolgedessen die Vollendung und Aufstellung der Statue ernsthaft gefährdet waren, muss hingegen offen bleiben.

Als feststand, dass Baccio Bandinelli die Neptunstatue nicht fertigstellen würde, gab die Republik ihr Vorhaben jedoch nicht auf, sondern wandte sich 1538 mit demselben Auftrag an einen anderen Künstler: Giovan Angelo da Montorsoli stellte Andrea Doria allerdings nicht in der Gestalt des Meeresgottes, sondern als antikisch gerüsteten Feldherrn dar, der über Türken und Waffengerät steht. ${ }^{53}$ Somit erschien der Großadmiral als überzeitlicher Tugendheld, dessen Darstellung in die Tradition antiker Herrscher- und Siegerbildnisse und damit in ein überpersönliches, politisch-moralisches Wertesystem eingebunden war. ${ }^{54}$ Im Gegensatz zur Neptunstatue von Baccio Bandinelli wurden im Standbild Montorsolis nicht vorrangig die Eigenschaften Andrea Dorias als friedvoller und mit Augenmaß agierender Herrscher betont, sondern dessen Sieg über die Osmanen. Dadurch wurde seine besondere Bedeutung sowohl für die Republik Genua als auch für die Verteidigung der christlichen Glaubenswelt im Mittelmeerraum herausgestellt - das Standbild von Montorsoli kann demnach auch als bildliche Entsprechung der im „Orlando Furioso“ begegnenden literarischen Stilisierung Andrea Dorias als Beschützer und bedeutender Feldherr verstanden werden. In der öffentlichen Darstellung des Genuesen trat somit die Betonung der guten Regierungsführung, wie sie in der imperialen Stoßrichtung der imitatio Neptuni aufschien, zugunsten der Akzentuierung von Sieg und Stärke zurück.

Bemerkenswert ist, dass einzelne bildliche Motive der imitatio Neptuni in die Darstellung des Großadmirals als Feldherr einfließen. Auf den Laschen des Panzerrocks der Statue Montorsolis, den sogenannten pteryges, sind neben Ornamenten und Tropaia Meereswesen zu erkennen, den Helm zu Füßen Andrea Dorias zieren Tritonen (Abb. 4, Abb. 5). Doch scheint auch umgekehrt der Gedanke, die Figuren

53 Zur Statue sowie ihrer Stellung im Oeuvre Montorsolis vgl. Laschke, Fra Giovan Angelo da Montorsoli (Anm. 3), S. 39-40, S. 150, S. 153, S. 160.

54 Laschke-Hubert, Quos ego oder wie der Meeresgott Neptun die Plätze eroberte (Anm. 3), S. 101. 


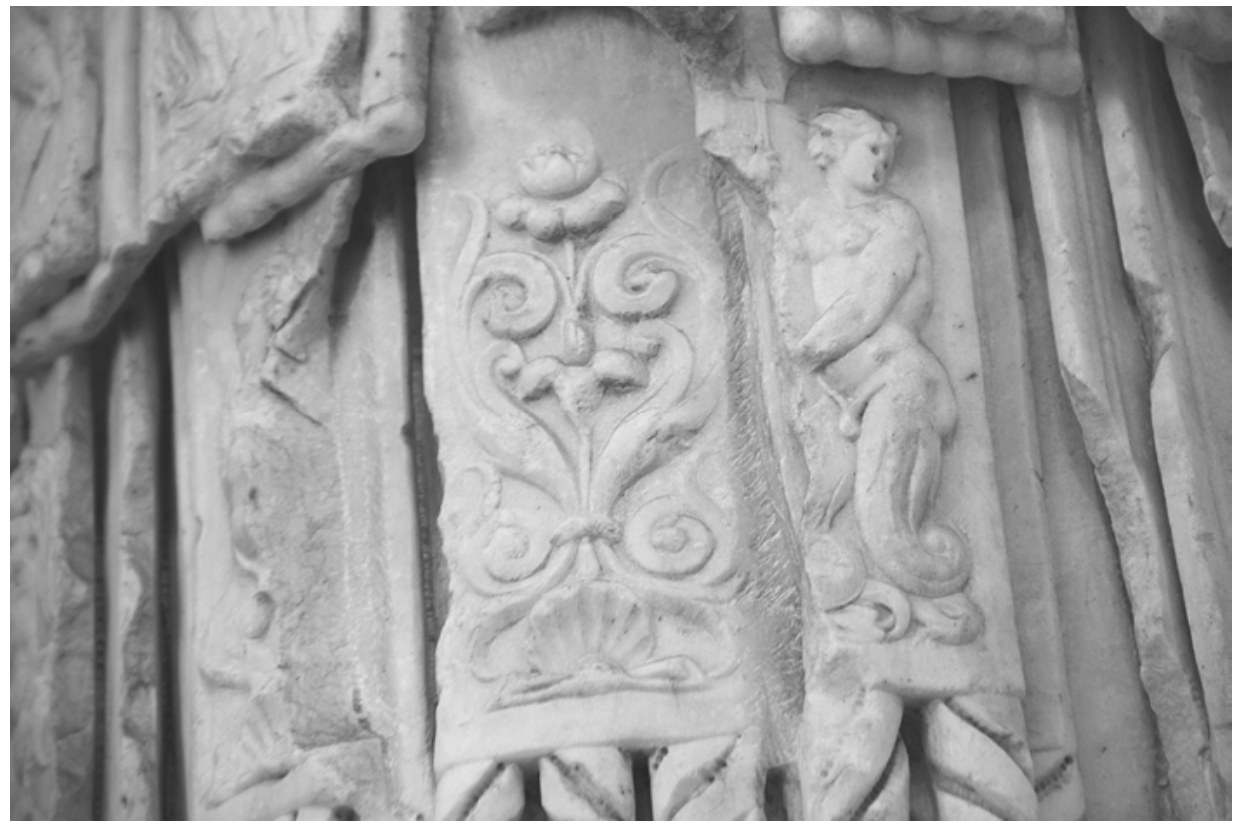

Abb. 4

Giovan Angelo da Montorsoli, Andrea Doria über Türken und Waffengerät, 1539-1540, Marmor, Genua, Palazzo Ducale, Detail der Pteryges

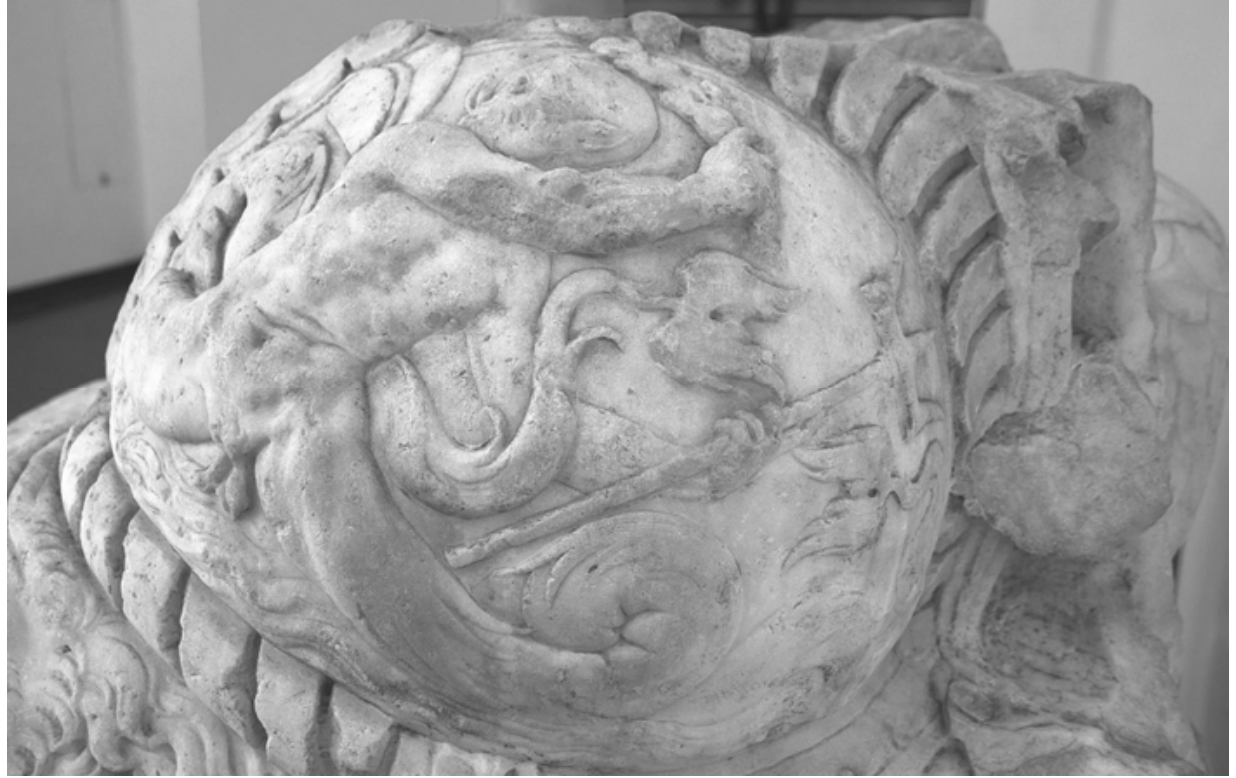

Abb. 5

Giovan Angelo da Montorsoli, Andrea Doria über Türken und Waffengerät, 1539-1540, Marmor, Genua, Palazzo Ducale, Detail des Helmes 
der besiegten Osmanen in die Konzeption der Sockelreliefs für das Neptunstandbild einzubinden, im Entwurfsprozess Baccio Bandinellis präsent gewesen zu sein. Besonders deutlich wird dies in einem vom 9. August 1537 datierenden Brief des Kardinals Hieronymo Doria, der in der Angelegenheit des Statuenprojektes für Andrea Doria als Stellvertreter der Republik Genua fungierte. Darin billigt er den offensichtlich vorangegangen Vorschlag Baccio Bandinellis, Figuren von ,Türken“ sowie andere Elemente in die Gestaltung des Standbildes einzubinden: „Quanto al mettere gli Turchi sotto gli piedi della statua, et far altri ornamenti, non lasciarete di mettere et aggiungere tutto quello vi parerà convenirsi ad una tale opera, che secondo la qualità di essa saranno riconosciute le vostre fatiche. " 55 Dieser Quelle lassen sich recht gut zwei im Louvre aufbewahrte Entwurfszeichnungen des Florentiner Bildhauers für das Sockelrelief zuordnen, die jeweils zwei unbekleidete und zum Teil gefesselte Männer zeigen. ${ }^{56}$

Es scheint, als sei mit der Darstellung Andrea Dorias als Feldherr eine für den republikanischen Kontext passendere Bildformel gefunden worden, um die Tugenden des pater et liberator patriae und dessen politische Rolle für sein Vaterland öffentlich zu veranschaulichen. ${ }^{57}$ Die als Freifigur gearbeitete Statue wurde zudem nicht, wie von Montorsoli intendiert, in der Mitte des Platzes vor dem Palazzo Ducale, sondern vor seiner Fassade aufgestellt. ${ }^{58}$ Dies lässt vermuten, dass die gesteigerte Bedeutung, die der Statue aus einer zentralen Position auf dem öffentlichen Platz erwachsen wäre, durchaus problematisch war und dass diesem Aspekt durch die Rückbindung an die Architektur des republikanischen Regierungssitzes entgegengewirkt werden sollte. ${ }^{59}$

55 Zitiert nach Waldman, Baccio Bandinelli (Anm. 12), Nr. 278, S. 164.

56 Auf die Zeichnung sowie deren mögliche Stellung im Entwurfsprozess des Standbildes von Baccio Bandinelli kann ich aus Platzgründen nicht eingehen. Auf den Zusammenhang zwischen dem zitierten Brief des Kardinals und den beiden Entwurfszeichnungen im Louvre hat bereits Fernando Loffredo hingewiesen, vgl. F. Loffredo, Baccio Bandinelli. Andrea Doria come Nettuno, in: D. Heikamp (Hrsg.), Baccio Bandinelli. Scultore e maestro (1493-1560) (Ausstellungskatalog Florenz), Florenz 2014, S. 575; zu den beiden Zeichnungen des Sockelreliefs vgl. Viatte, Inventaire général des dessins italiens / Musée du Louvre (Anm. 24), Nr. 51-52, S. 162-163, dort auch mit weiterer Literatur.

57 Zur Bedeutung der Statue Montorsolis als exemplum für die Republik Genua vgl. Boccardo, Andrea Doria e le arti (Anm. 3), S. 115; Polleroß, Rector Marium or Pater Patriae? (Anm. 23), S. 112-113.

58 Nach Vasaris Angaben in Montorsolis Biographie sollte die Statue ursprünglich auf der Piazza Doria aufgestellt werden, womit der kleine Platz vor der Familienkirche gemeint gewesen sein müsste, der von zahlreichen, den Doria gehörenden Palazzi umstanden war, vgl. Laschke-Hubert, Quos ego oder wie der Meeresgott Neptun die Plätze eroberte (Anm. 3), S. 101. Zur Aufstellung der Statue Montorsolis vor der Fassade des Palazzo Ducale, die jedoch dem Innenhof zugewandt war, vgl. S. Hanke, „Più libero di qualsivoglia altro luogo.“ Die Piazza Banchi in Genua, in: A. Nova / C. Jöchner (Hrsg.), Platz und Territorium. Urbane Struktur gestaltet politische Räume, München 2010, S. 197-222.

59 Laschke-Hubert, Quos ego oder wie der Meeresgott Neptun die Plätze eroberte (Anm. 3), S. 101; Hanke, Die Macht der Giganten (Anm. 11), S. 165-186. 
Letzten Endes beanspruchte Andrea Doria die Figur Neptuns für die skulpturale Ausstattung des Gartens seines Palazzos in Fassolo. Nachdem das Projekt der ersten Statue gescheitert war und Baccio Bandinelli den unvollendeten Marmorblock in Carrara liegen gelassen hatte, beauftragte er eben jenen Montorsoli, auf dem höchstgelegenen Punkt eines ansteigenden Hügels im Garten seines direkt am Meer gelegenen Palazzos eine überlebensgroße Neptunstatue aus Stuck aufzustellen. ${ }^{60}$ Die monumentale bildliche Assoziierung des Genuesen mit dem Meeresgott blieb somit zwar auf den halböffentlichen Raum der Residenz des Großadmirals beschränkt, war innerhalb der Republik jedoch weiterhin präsent.

\section{Abbildungsnacbreise}

Abb. 1, 2, 4, 5 Katharina Helm.

Abb. 3

(C) Trustees of the British Museum.

Farbabb. 2 Nach C. B. Strehlke (Hrsg.), Pontormo, Bronzino, and the Medici. The Transformation of the Renaissance Portrait in Florence (Ausstellungskatalog Philadelphia), Philadelphia 2004.

60 Die Stuckstatue ist nicht erhalten, da sie vermutlich 1586 durch eine acht Meter große Jupiterstatue Marcello Sparzios ersetzt worden war. Ob sie die Gesichtszüge Andrea Dorias getragen hat, muss daher hypothetisch bleiben, vgl. Laschke-Hubert, Quos ego oder wie der Meeresgott Neptun die Plätze eroberte (Anm. 3), S. 101-102. Zum Anblick der Gartenanlage und ihres Skulpturenschmucks vom Hafen aus vgl. Hanke, Die Macht der Giganten (Anm. 11), S. 169. 